\title{
Deployment Verification of Large CFRP Helical High- Gain Antenna for AIS Signals
}

\author{
Tom Sproewitz \\ German Aerospace Center \\ Robert-Hooke-Str. 7 \\ D-28359 Bremen \\ +4942124420237 \\ tom.sproewitz@dlr.de
}

\author{
Joachim Block \\ German Aerospace Center \\ Lilienthalplatz 7 \\ D-38108 Braunschweig \\ +495312952387 \\ joachim.block@dlr.de
}

\author{
Annette Bäger, Lars Hauer \\ German Aerospace Center \\ Robert-Hooke-Str. 7 \\ D-28359 Bremen \\ +4942124420237
}

\author{
Martin Schuetze \\ Schuetze-Staebe GmbH \\ Burenkamp 10 \\ D-46286 Dorsten \\ +49236922439
}

Abstract - In this paper the development of the structural design of a deployable helical antenna made from fiber composite material as well as its deployment verification in Zero-G environment will presented ${ }^{1,2}$. In the introduction the advantages of helical antennas will be pointed out and a potential field of application, the receiving of AIS (Automatic Identification System) signals from maritime vessels, will be presented. The next chapter deals with the antenna design where especially manufacturing aspects will be addressed. The test setup for deployment tests in weightlessness will be explained and the results recorded during the $15^{\text {th }}$ parabolic flight campaign (PFC) of DLR (German Aerospace Center) in March 2010 will be shown. During this campaign the deployment of 4 different helix antennas was tested as well as reaction forces and the dynamical behavior were recorded. An outlook is given on the subsequent finite element (FE) nonlinear numerical analysis. The aim of these calculations is to correlate analysis and test results, to use the correlated models for further improvements of antenna parameters, and to enhance predictions of the antenna behavior and its effect on the satellites attitude control during and after deployment.-

\section{TABLE OF CONTENTS}

1. INTRODUCTION

2. HeLiX ANTENNA DESIGN

3. DEPLOYMENT TEST SET-UP

4. DEPLOYMENT TEST RESULTS.

5. DEPLOYMENT NuMERICAL SIMULATION

6. SUMMARY / CONCLUSIONS .............................................11

REFERENCES ...................................................................11

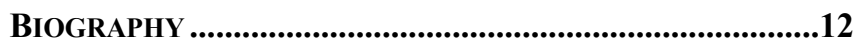

\section{INTRODUCTION}

Helical antennas offer some superior properties compared to other antenna types $[1,2]$. They are very well suited for transmitting and receiving circularly polarised electromagnetic waves, and they have an excellent directionality. Their gain increases with the number of windings, for at least up to 7 or 8 windings. Together with the rise in the achievable gain, the footprint of the antenna

\footnotetext{
${ }^{1}$ 978-1-4244-7351-9/11/\$26.00 (2011 IEEE

${ }^{2}$ IEEEAC paper\#1568, Version 4, Updated 2011:01:12
}

gets smaller. To have a characteristic which may point towards one specific direction the antenna also needs a conductive plate as a reflector.

Based on these characteristics helical antennas are, besides many other applications, very well suited for space applications where a comparably small footprint area on ground is required and a high antenna gain due to low signal strengths is needed.

In the past years a rising interest in receiving of AIS signals of maritime vessels from space could be noticed. The AIS system has been introduced to improve the security and to simplify the control of the seafare trade. Each individual ship constantly sends data like identity, position, heading, speed etc. which basically serves as an anti collisioning system. AIS signals are transmitted and received at a frequency of either $161.975 \mathrm{MHz}$ (AIS channel A / channel 87B) or $162.025 \mathrm{MHz}$ (AIS channel B / channel $88 \mathrm{~B}$ ) in the VHF (Very High Frequency) range. This works very well in eyeshot distance between shore stations and vessels or between vessels itself. But in order to cover larger areas or even to provide worldwide data of ship movements the application on a satellite seems to be a very good opportunity.

The communication between ships on water works very well. Each ship knows all other ships in its vicinity and the AIS system arranges time slots for each ship to send the AIS data. When receiving AIS signals from space and hence covering a large area with high ship traffic, the satellite will receive data from ships that are not synchronised with each other. This will lead to overlapping signals, which may be decoded only partially, if at all. The direct result may be the impossibility of detecting ships in certain areas as has already been happened in regions with high ship traffic like the east coast of North America or the German Bay.

In order to increase the resolution of signals when received in space, a helix antenna could be a very good application. It reduces the footprint size and thus the number of signals that may collide. Furthermore the strength of the received signals is much higher compared to signals which are received by conventional bar antennas. A good example is the AISat project which will be explained below. The innovative antenna concept which has been developed for 
this project could easily be transferred also to other small satellites in the future.

\section{AISat Project}

AISat is a nanosatellite with a $4 \mathrm{~m}$ long helical high-gain antenna (see Fig. 1). The excellent directionality of the helical antenna shall allow to improve the signal quality and to reduce the risk of overlapping signals. Up to now this was not possible: Firstly, satellites usually receive signals from a much larger area, and secondly, many interfering signals are present in the frequency bands of interest. Besides AIS the antenna shall also enable the receiving of SAR (Search and Rescue) signals sent from survival crafts or distressed vessels. AISat will operate from a sunsynchroneous orbit with approx. $650 \mathrm{~km}$ altitude at 10:00 LTDN (Local Time Descending Node). Its launch is scheduled for August 2011 on a PSLV (Polar Satellite Launch Vehicle) from the Satish Dhawan Space Centre on the Island of Sriharikota, India.

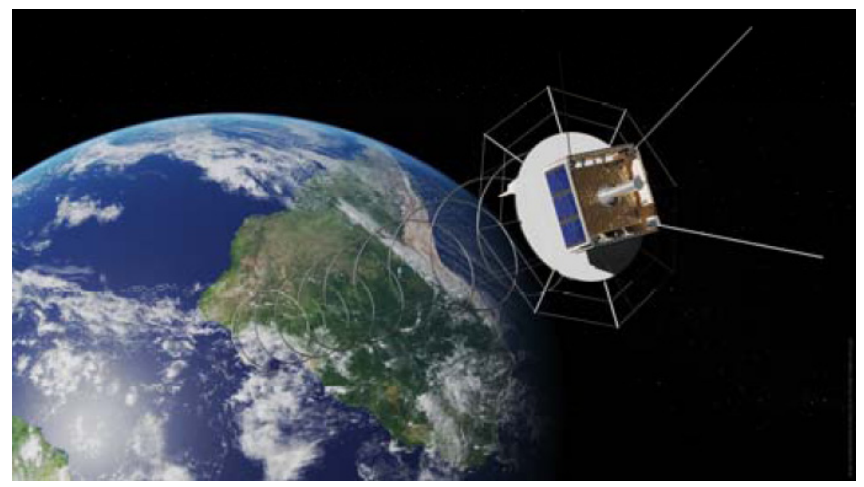

Figure 1 - AISat in Orbit (Artist's View)

All the superior electromagnetic properties of helical antennas can be combined with the mechanical advantage that the antenna can be used as a compression spring. This requires that its core structure has sufficiently high spring stiffness but can nevertheless easily be compressed. The need of compressing the antenna comes from its application on the AISat where its stowed height must not be larger than $100 \mathrm{~mm}$. Such an antenna has been developed by DLR Institutes in Bremen and Braunschweig together some industrial partners. The development was very challenging since the antenna has to expand from a stowed stack length of the above mentioned $100 \mathrm{~mm}$ to a total length of $4 \mathrm{~m}$. Only a special fiber composite core under a conductive coating and a system of stabilizing cords led to a satisfying solution.

Both the self-deployment and the self-stabilization function of this innovative antenna concept have been successfully tested and verified under zero-g-conditions during the $15^{\text {th }}$ PFC of DLR. It could be demonstrated that the helical antenna can achieve its desired contour in weightlessness within some seconds and maintain the required stability.
Beyond the current application for the AISat satellite it is therefore a quite promising concept for future satellites.

\section{Antenna Concept Selection for AISat}

As already mentioned the helical antenna on the nanosatellite must be stowable to a very small volume during launch (see Fig. 2). Therefore, the predefined helical geometry shall also be used for a second objective, as compression spring for self-deployment. Once in orbit, the spring will be released and will immediately self-deploy until it has reached its full operational length. To ensure a stable and exactly defined final position, thin control cords limit the further expansion against a prior chosen residual spring force (Fig. 3).

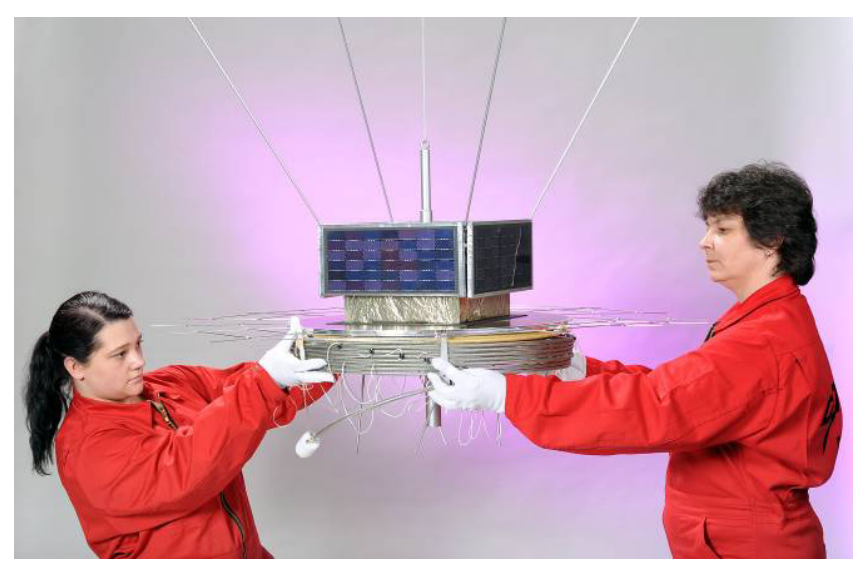

Figure 2 - AISat Breadboard Model (stowed)

The chronological separation of mechanical and electrical functions (first deployment, second antenna operation) suggests a physical separation of the antenna materials as well:

1. the antenna core needs a high spring stiffness but no electrical conductivity

2. the outer skin needs an excellent electrical conductivity, but no mechanical stiffness.

Therefore it was decided to cover the core with a thin metallic fabric hose consisting of tinned copper wires. The core itself is a cylindrical sandwich strut consisting of a hard foam core and fiber fabric hose. Depending on the required spring stiffness materials of interest would preferably be carbonfibers, but glassfibers or aramid fibers could alternatively be selected.

\section{Partners}

AISat is a DLR internal project where basically two institutes are involved. The Institute of Space Systems in Bremen developed the main body of the nanosatellite and the antenna base structure and it performed the whole system engineering. The Institute of Composite Structures and Adaptive Systems in Braunschweig was responsible for 
the experimental verification of the deployment of the helical antenna. The Schütze-Stäbe $\mathrm{GmbH}$ Company in Dorsten performed the manufacturing of the antenna hardware and corresponding material creep tests. In terms of the electrical design of bus and antenna, DLR's key partners are the Bremen University of Applied Sciences and the consulting company Joachims HF\&EDV-Beratungs $\mathrm{GmbH}$.

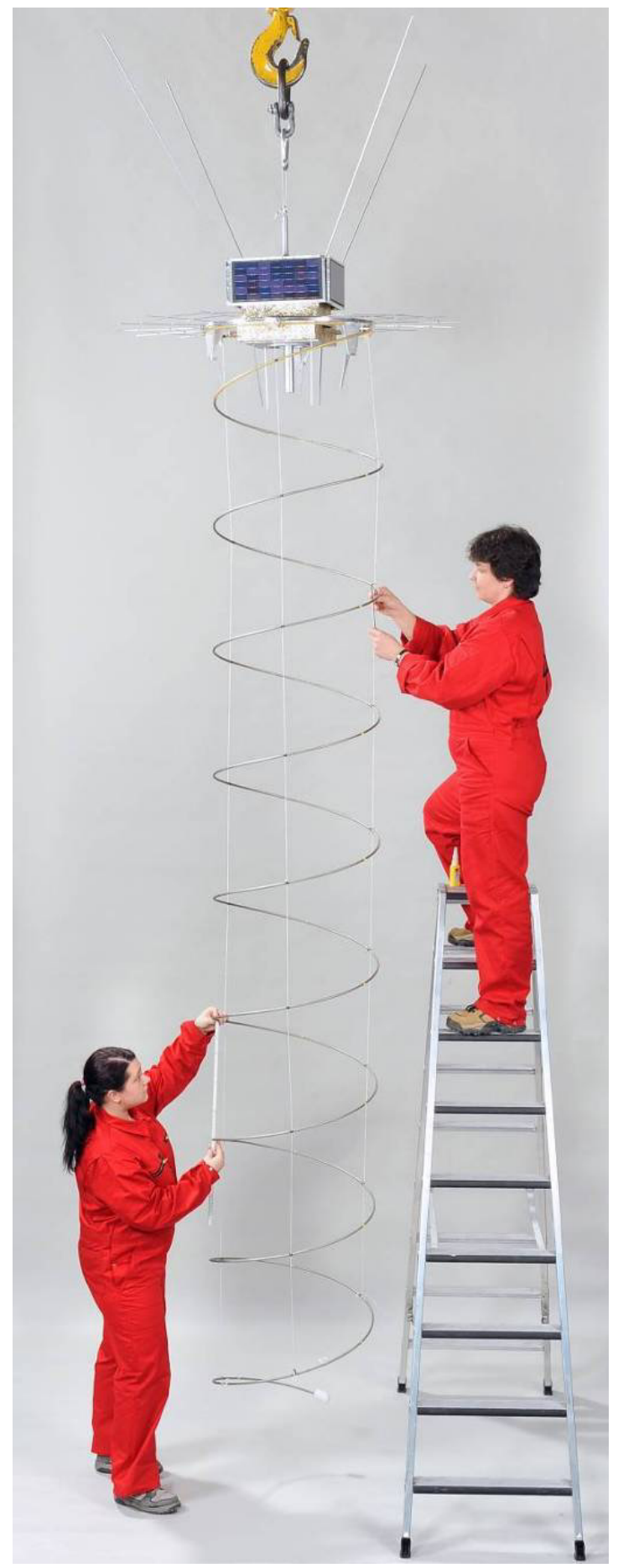

Figure 3 - AISat Breadboard Model (deployed)

\section{HeliX Antenna DeSIGn}

\section{Existing Helical Antenna Design for Satellites}

Helix antennas have already been used for space applications. But depending on their field of application their design can be very different. Large helix antennas have already been flown on satellites (see Fig. 4) with an undeployable design and hence with a very robust backing structure. If such antennas would have to be deployable a lot of mass had to be added for complex mechanisms. Due to new radio frequencies of new satellites the size of the antennas decreased. Comparably small antennas are rigidly mounted either in a housing or totally exposed to space.

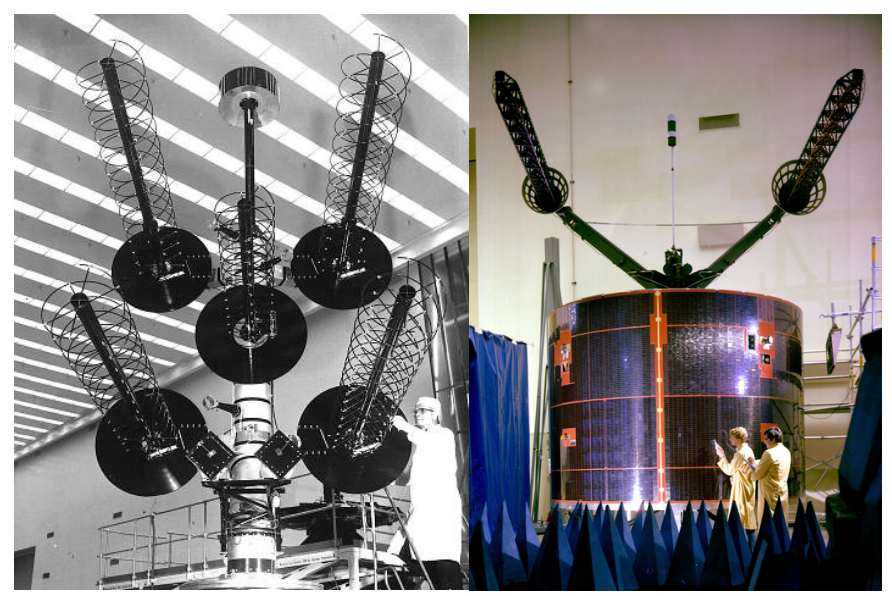

Figure 4 - TACSAT-1 (1969) and LEASAT (1998)

Several patents exist on deployable helix antennas. In the US patents $[3,4,5]$ the deployment of helix antennas with multiple wires is described. All of them require a torsion of the antenna wires about the antenna longitudinal axis. Some of them are also motor driven, which require additional

movable parts and electrical power. The patents $[6,7,8]$ show single wire helix antennas which are partially selfdeploying. The structures shown in [6,7] have a quite complicated strut framework to support and deploy the antenna which could easily loose its proper function in case of small damages. Patent [8] shows a very simple spiral spring like antenna, which will deploy due to formerly stored potential energy during stowing. The spring material is an electrically conducting material which also serves as the actual antenna. Piecewise solid segments ensure a proper alignment in length and in lateral direction (see Fig. 5). 


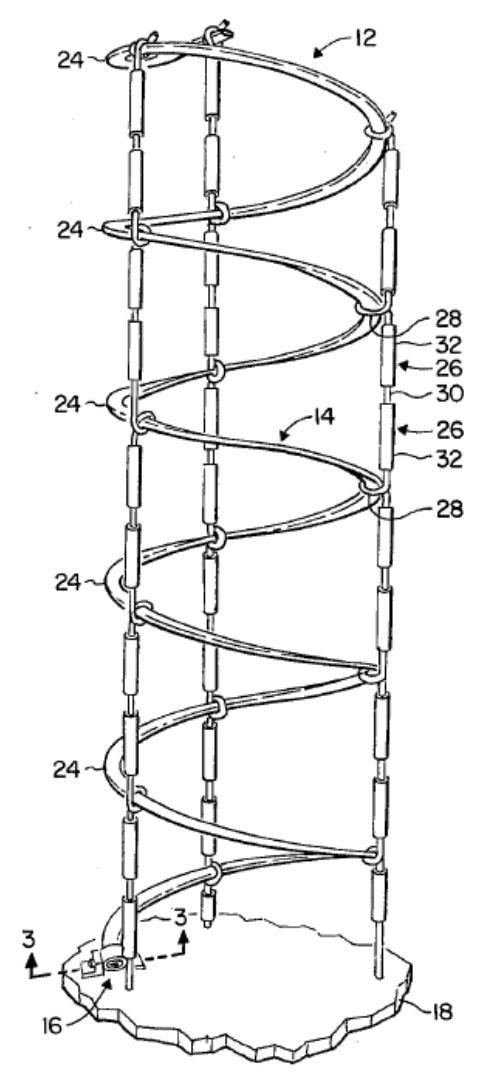

\section{Figure 5 - Deployable Helix Structure (US Patent 4068238)}

\section{Envisaged Helical Antenna Design}

As mentioned earlier, the AISat helix antenna is made of a sandwich strut consisting of a hard foam core and a braided fiber composite sleeving as depicted in Fig. 6. The length of the antenna is controlled by means of three flexible control cords. These are braided glass-fiber cords with a diameter of $1 \mathrm{~mm}$ made from SILTEX E-glass material [9] (see Fig. 3). Since fiber composites are bad electric conductors the strut is additionally covered with a metal fabric. More details on the design follow hereafter.

\section{Primary Composition of Helix Wire}

For the manufacturing of the helix wire a (carbon/aramid)fiber braided sleeving (Fig. 6, item (2) is pulled upon a cylindrical hard foam core (Fig. 6, item (3)). The fibers are impregnated with epoxy resin and then surrounded with a thin heat shrink tube. This process is almost identical with the well-proven manufacturing process of straight pultruted carbonfiber struts [10], apart from two differences:

1. The shrinkage hose consists of PE (polyethylene) instead of glassfibers.
2. There are no unidirectional fibers in longitudinal direction, because they would inevitably tend to buckle on the inner side of the windings (which cannot occur on straight struts).

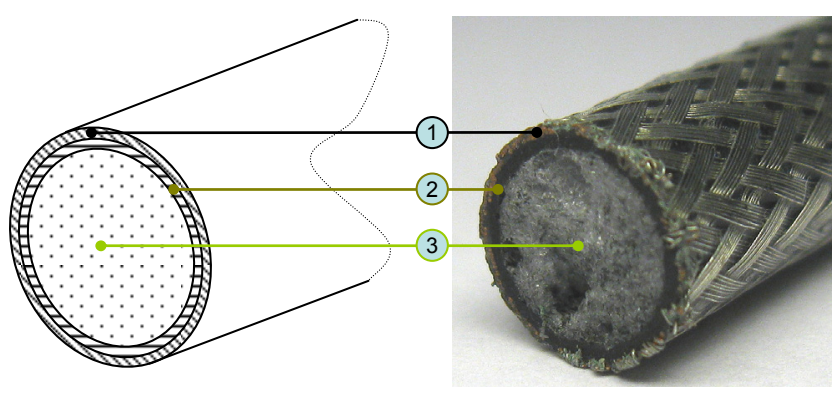

Figure 6 - Composition of Helix-Wire Material

Another reason for the usage of a braided fabric sleeving without unidirectional fibers lies in the fact that the helix will be stowed like a spiral spring. In order to reach a high spring stiffness in a spiral spring, the shear modulus is the most critical material parameter due to slight torsion during spring compression.

$$
F_{\text {Spring }}=\frac{G d^{4}(L-n d)}{8(D-d)^{3} n}
$$

With: $\quad G$-shear modulus

$$
\begin{aligned}
& \mathrm{d} \text { - spring wire diameter } \\
& \mathrm{L} \text { - free length of spring } \\
& \mathrm{n} \text { - number of active windings } \\
& \mathrm{v} \text { - Poisson ratio } \\
& \text { D - spring outer diameter }
\end{aligned}
$$

To ensure a high capability of bearing shear loads induced by torsion the fabric hose consists of rovings which are oriented in $\pm 45^{\circ}$ when applied to a strut with nominal diameter. In case of the helical antenna with diameters between $8.5 \mathrm{~mm}$ and $9.5 \mathrm{~mm}$ orientations of about $\pm 30^{\circ}$ could be achieved.

The CFRP (Carbon Fiber Reinforced Plastic) helix wire is made of a Torayca T300 [11] braided sleeving from SILTEX. The fibers of the helix with an aramid braided sleeving are conventional aramid fibers which have not been distinctively identified by the sleeving manufacturer SILTEX. A VETROTEX E-glass EC11 136 Z28 [12] braided sleeving from SILTEX is used for the CFRP helix inlet. As matrix system for all different composite helix wires the Scheufler epoxy matrix system L160/H163 [13] has been used. 


\section{Setting up Helix Structure}

In a second step, sandwich structure with unhardened resin is wound upon a cylindrical mould which reflects the geometry of the helical antenna (see Fig. 7). This is the most critical step during the helix manufacturing, because several shrinkage and creep effects have to be taken into account in advance. In longitudinal direction, the overall length of the helix must be significantly larger during manufacturing (i.e. during the winding upon the cylindrical form) than in the nominal design case. This is necessary to ensure a determined residual spring force for additional structural stiffness after the antenna has reached its length of $4 \mathrm{~m}$ during the deployment process. In radial direction, it must be considered that any elongations and shortenings of the helix will simultaneously influence the winding radius.

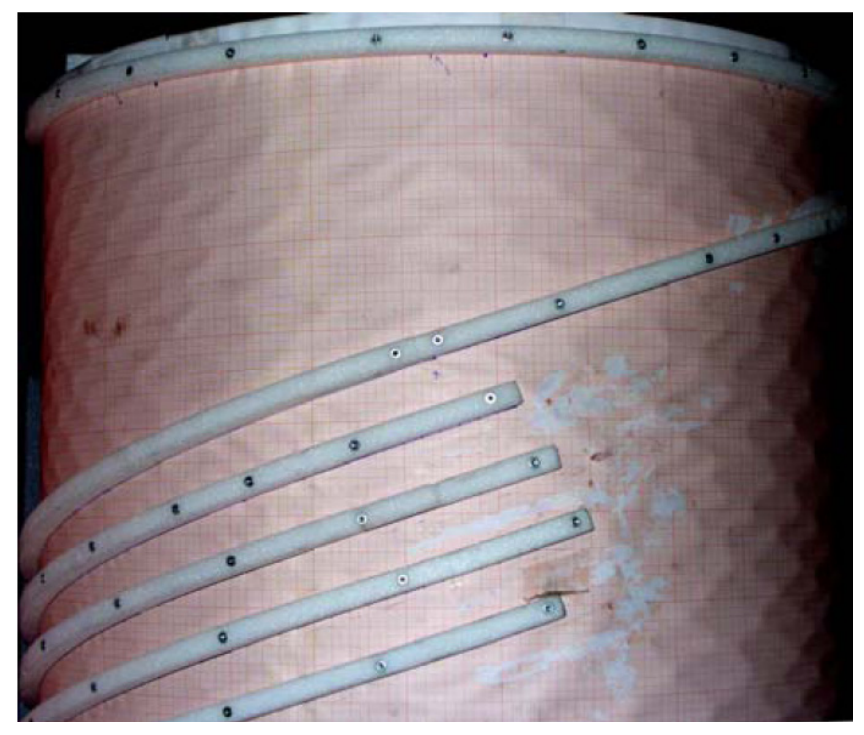

Figure 7 - Mould for Helix-Manufacturing

Once the desired contour has been fixed on the cylindrical form, the whole helix is hardened and cured at elevated temperatures $\left(+60^{\circ} \mathrm{C}\right.$ and $\left.+80^{\circ} \mathrm{C}\right)$. But even after curing, the structure remains subject to creep effects: Long-term stowage in compressed configuration will reduce the overall length and only after longer relaxation periods the material will slowly "regenerate" (Fig. 8). But the structures made from aramid (red) show much higher creep effects than the CFRP structures. This is essential information for the selection of a suitable material for an in-orbit application.

To avoid a changing antenna performance it is mandatory to ensure a deployed length of $4 \mathrm{~m}$ at any time in the orbit even though the antenna has been stowed for a certain amount of time. Therefore the manufacturing length of the antenna is envisaged to be $6 \mathrm{~m}$ (4.2 $\mathrm{m}$ for PFC structures) with a reduced diameter to have nominal diameter values when pre-stressed to envisaged length. Three control cords will be used to hold the antenna at its nominal length and to ensure a proper alignment, which will be discussed below. Furthermore it is part of the integration procedure to store the antenna as long as possible in undeformed configuration.

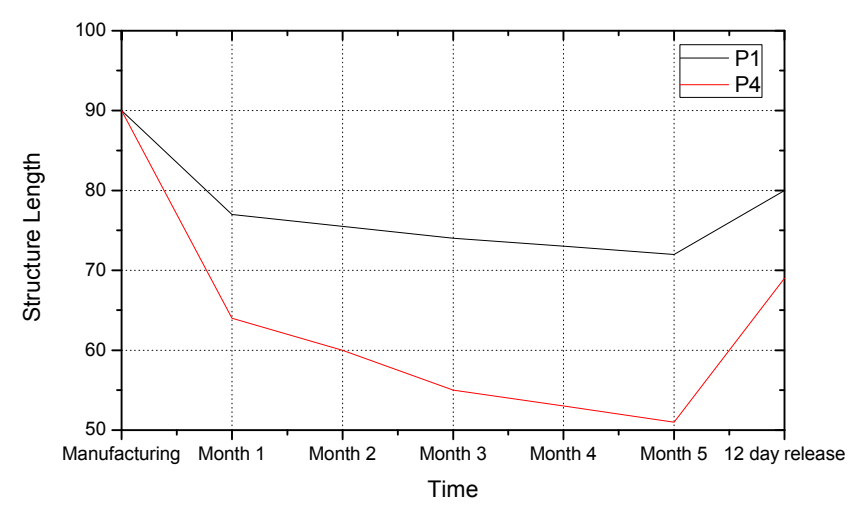

Figure 8 - Antenna Length during long term
Stowage and Relaxation

Application of electrically conductive Coating

After demoulding the hardened and cured fiber composite helix structure is covered with the metallic fabric hose (Fig. 6, item (1). At the root of the first antenna winding, the antenna inlet, there should remain an electric "gap", i.e. after mounting on the reflector plate there should be no conductive connection. Instead, the metallic fabric hose will begin with a certain offset above the reflector plate and will be connected to the electric circuitry on board the satellite by a suited cable.

\section{Application of Control Cords}

The creep and relaxation effects described above make it inevitable to manufacture the helices with sufficient additional length as mentioned above, since it must be secured that:

1. the nominal operational dimensions are achieved after deployment in space under all circumstances.

2. the structure has a sufficiently high pre-stressing to increase the overall stiffness and hence the dynamical behavior of the helix antenna structure.

Therefore the manufacturing length, as mentioned above, is chosen to be significantly larger than the final nominal length of $4 \mathrm{~m}$ and the antenna has to be fixed at nominal length when it is deployed. This means that in longitudinal direction three electrically isolating control cords with an angular distance of $120^{\circ}$ between each are required. Additionally these cords also have to counteract the residual spring force and to align the deployed antenna very accurately. The control cords are fastened on precisely measured points on the top winding of the helix and, with their other end, on the reflector plate. At all cross points with windings other than on top and bottom, the control cords are located in slip rings to enable relative movements between the cords and the helix structure itself. See Fig. 9 
for the principle of fixation and bearing between helix wire and control cords.

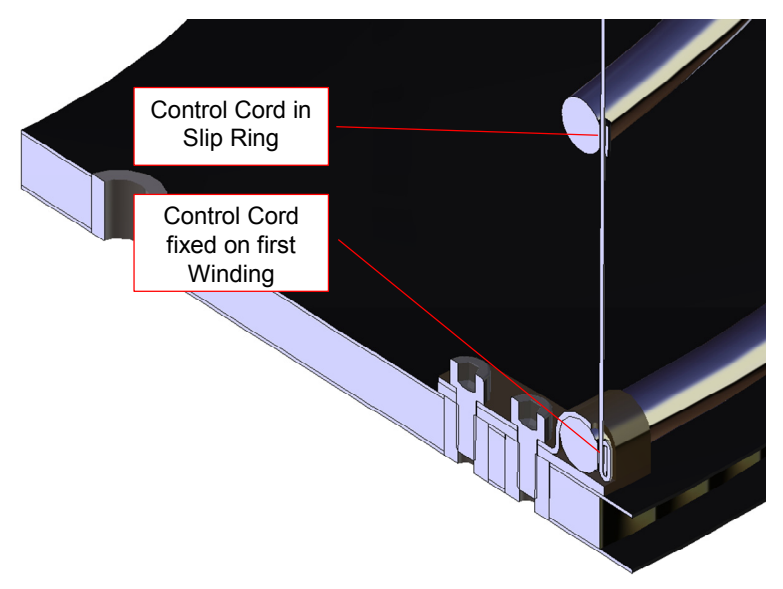

Figure 9 - Fixation and Slip Ring Bearing between Helix Wire and Control Cords

See Fig. 10 for a deployed structure with integrated dummy reflector plate and control cords.

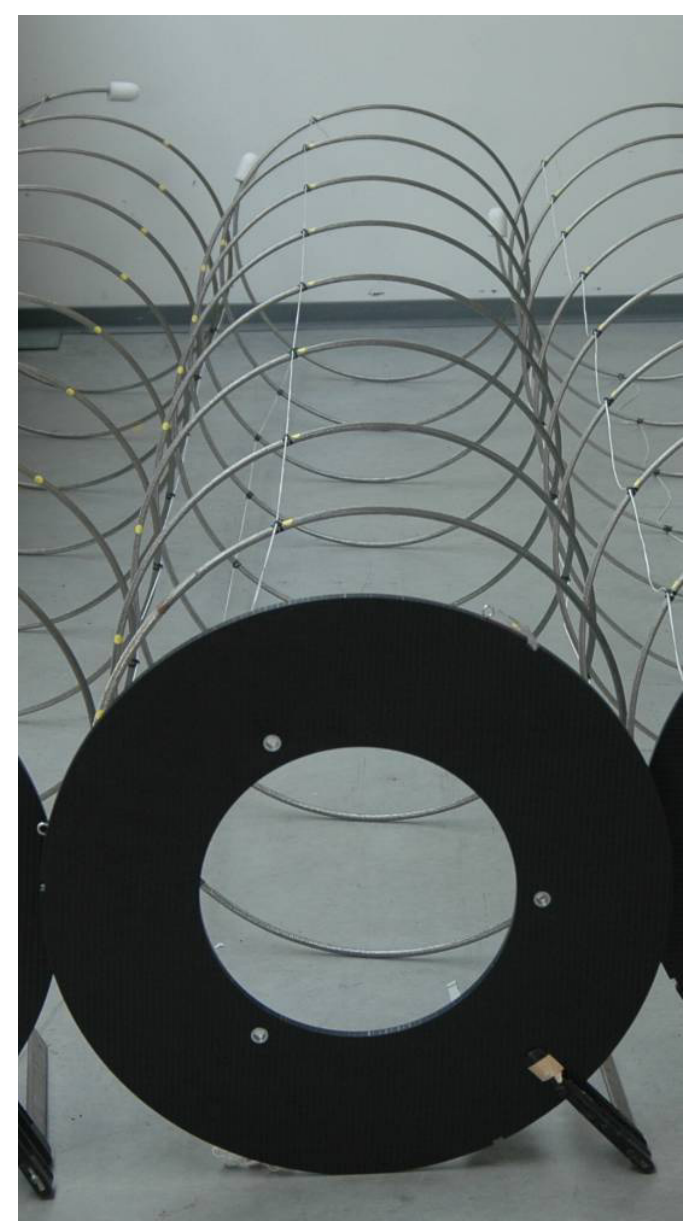

Figure 10 - Helix for Parabola Flight (deployed) with Control Cords

\section{DEPLOYMENT TEST SET-UP}

\section{Justification for Parabola Flight and Aims}

A deployment of the structure as the helical antenna described above could never be tested satisfactorily under normal gravity. Apart from all other disturbing effects, the antenna would already be deflected downwards by its own weight immediately after release. Even with gravity compensation devices it would be almost impossible to compensate gravity and simultaneously allow for contraction, bending and torsion. Also, the antenna could never be installed on AISat (or on any other satellite) without having been thoroughly tested and qualified. Therefore it became mandatory to test the release function within a parabolic flight where up to 22 seconds of weightlessness per parabola are available, to:

- monitor the self-deployment and demonstrate a faultless operation

- verify the achievement of a well-aligned final attitude in space

- demonstrate that dynamical structure deflections will noticeably decay in less than $20 \mathrm{sec}$

- measure and record reaction forces during and right after deployment for satellite attitude control.

During the one-day parabolic flight campaign 31 parabolas were performed during the flight. They are divided into 6 series of 5 parabolas each, plus one initial parabola to become familiar with the procedure. Table 1 summarizes the test plan for the AISat antenna.

\section{Test Structure Description}

Since the helix stiffness is the most critical parameter for the deployment behavior, four different structures with varying wire material and wire cross section were tested during the PFC.

As listed in Table 1, the helix structures $\mathrm{P} 1$ and $\mathrm{P} 3$ are made from CFRP with a GFRP (Glass Fiber Reinforced Plastic) inlet. Both structures vary in cross section with P1 having $8.5 \mathrm{~mm}$ wire diameter and $\mathrm{P} 3$ having $9.5 \mathrm{~mm}$ diameter. P2 and P4 are completely made of aramid fiber reinforced plastic with P2 having $8.5 \mathrm{~mm}$ diameter and P4 having $9.5 \mathrm{~mm}$ diameter. The GFRP inlet for P1 and P3, on which the helix structure is fixed to the reflector plate, had to be foreseen since CFRP is slightly electrically conductive. If the whole structure had been made from CFRP, a weak electrical link between the end of the metal fabric hose and the reflector plate would be possible but would lead to gain losses or even to a short circuit.

A whole test structure consists of the before described helix structure including its control cords, a ground plate 
(reflector plate on the satellite) as well as hold-down \& release mechanisms. In Fig. 11 there is shown a stowed helix structure mounted on the test rack including all above mentioned structural parts.

Table 1 - Parameters and Test Sequence of Test Structures

\begin{tabular}{c|c|c|c|c|c}
\hline $\begin{array}{c}\text { Parabola } \\
\text { Sequence }\end{array}$ & Label & Material & $\begin{array}{c}\text { Wire } \\
\text { Diameter }\end{array}$ & Mass & $\begin{array}{c}\text { Prestressed } \\
\text { Length }\end{array}$ \\
\hline 1 & P1 & $\begin{array}{c}\text { CFRP/GFR } \\
\text { P }\end{array}$ & $8.5 \mathrm{~mm}$ & $0.86 \mathrm{~kg}$ & $4.0 \mathrm{~m}$ \\
\hline 2 & $\mathrm{P} 2$ & Aramid & $8.5 \mathrm{~mm}$ & $0.73 \mathrm{~kg}$ & $4.0 \mathrm{~m}$ \\
\hline 3 & $\mathrm{P} 3$ & $\begin{array}{c}\text { CFR/GFR } \\
\mathrm{P}\end{array}$ & $9.5 \mathrm{~mm}$ & $0.92 \mathrm{~kg}$ & $4.0 \mathrm{~m}$ \\
\hline 4 & $\mathrm{P} 4$ & Aramid & $9.5 \mathrm{~mm}$ & $0.88 \mathrm{~kg}$ & $4.0 \mathrm{~m}$ \\
\hline 5 & $\mathrm{P} 1$ & $\begin{array}{c}\text { CFRP/GFR } \\
\text { P }\end{array}$ & $8.5 \mathrm{~mm}$ & $0.86 \mathrm{~kg}$ & $3.5 \mathrm{~m}$ \\
\hline 6 & \multicolumn{5}{c}{ Photographic Documentation } \\
\hline
\end{tabular}

The ground plate is a sandwich plate made from CFRP face sheets and an Al-honeycomb core. A number of inserts is implemented to attach fixation clamps, hold-down \& release mechanisms and to foresee interface points for the attachment to the test rack.

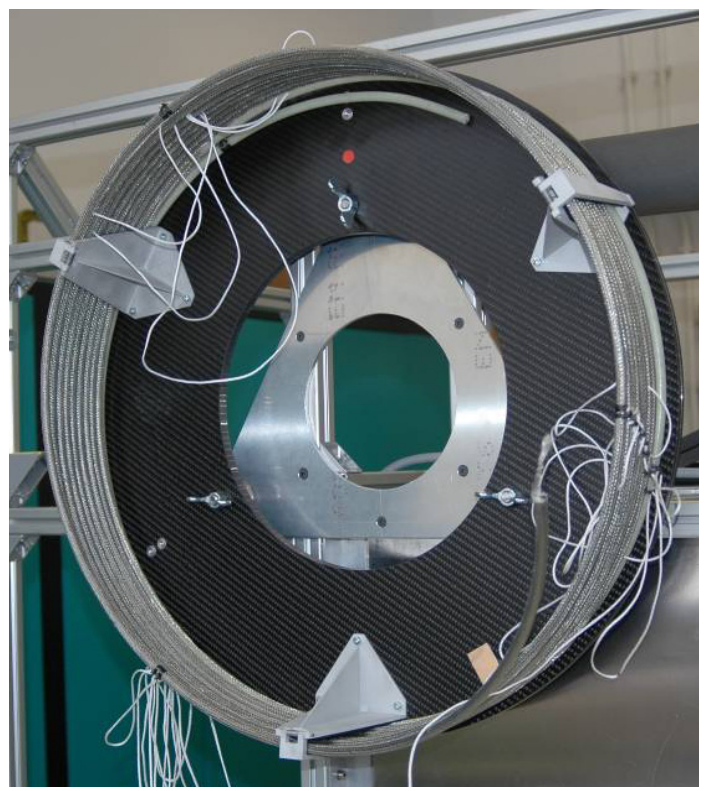

Figure 11 - Helix for Parabola Flight (stowed) with Ground Plate and Mechanisms

Three CFRP fixation clamps will rigidly connect the helix structure to the ground plate. The three hold-down \& release mechanisms have to ensure a save mounting of the stowed antenna during launch and a save release (see Fig. 11). They consist of a mechanism base and a rotatable arm which will automatically open the mechanism by means of two small leg springs.
The three hold-down \& release mechanisms are manually triggered upon injection into the zero-g phase by pulling a pin to release the circumferential helix release cord which fixes the hold-down arms in its closed configuration (Fig. 12). The manual triggering was preferred in order to avoid any control electronics. On the satellite structure in space the circumferential string will be cut by means of a melting element. Once released, the antennas should safely unfold up to a total length of $\approx 4 \mathrm{~m}$, with a helix diameter of $0.57 \mathrm{~m}$.

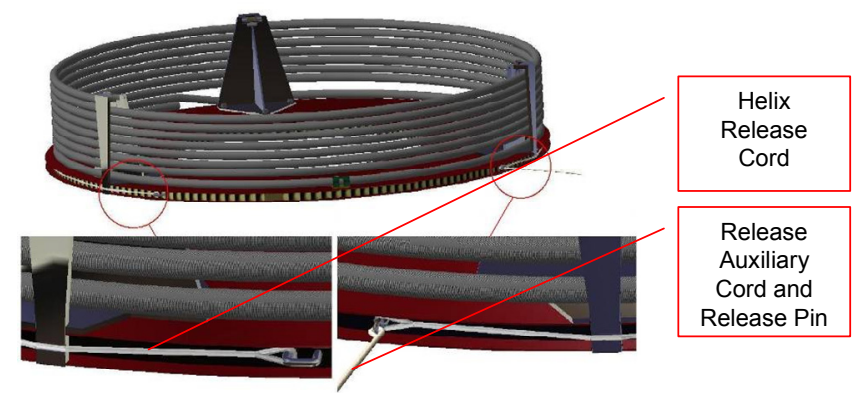

Figure 12 - Circumferential Helix Release Cord

\section{Test Rack Description}

The helix structures were stored in stowed configuration in a magazine box on the test rack. For each test series (5 parabolas) one of them was taken out and locked in vertical position on a triangular interface plate with integrated force transducers so that the reaction forces upon deployment could be measured (Fig. 13). The measurement equipment consists of:

- three 3-axial preloaded force transducers

- $\quad$ three charge amplifiers

- two dynamic signal analysers

- one laptop with data acquisition software.

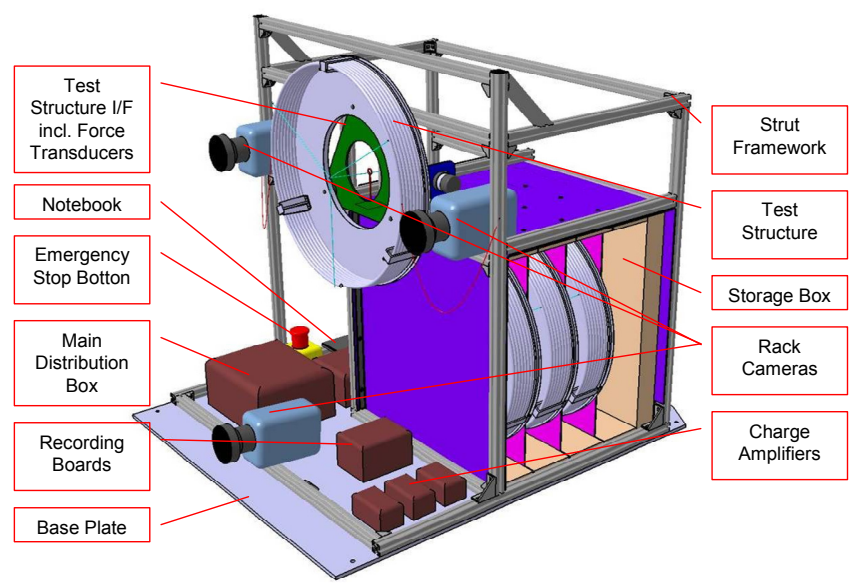

Figure 13 - Parabolic Flight Test Rack 
For the recording of the unfolding process three video cameras were fastened on the test rack and two additional cameras mounted on the aircraft handrail behind and in front of the test rack. All cameras were self-recording. They were switched on prior the first parabola and switched off after the last parabola. In order to be able to correlate the aircraft zero-g data with the test results the clock of the recording laptop and the system clock of the aircraft were synchronized. To correlate the movie material recorded by the video cameras the trigger signal for the start of the recording of the force transducer data has been used to give a light signal to all cameras.

\section{Deployment Test Results}

It could successfully be verified that within the 22 seconds of zero-g conditions in each parabola the helical antenna structure can be completely deployed to its full length (see Fig. 14). It could further be verified that the structural damping is high enough to reduce motions in the time between deployment and the end of zero-g such that almost stable conditions are reached.

The presence of the control cords leads to strong vibrations induced by the shock loads when the cords are tightened. Further vibrations and reaction forces are induced by the rebouncing of the helix structure and the corresponding bending and torsion motions. However, these motions are well damped due to comparably high structural damping of the sandwich struts and the friction between the helix wire and the control cord as the structure is in motion.

Concerning the directionality of the forward motion, it was observed that the antenna structures did not exactly deploy along their axis of symmetry. If the initial expansion is misaligned then the points in time when each of the three control cords reaches its final length will strongly differ, so that the antenna rebounds in an asynchronous manner, and the vibrations and reaction forces become worse.

For all tests all antenna structures deployed in the same direction. This led to the conclusion that there are the same reasons and that these reasons do not have a random nature. There are mainly two reasons that could be identified causing the effect of misaligned deployment:

1. Friction between helix wire and the flanges of the release mechanisms as well as the relative position of the helix inlet to these flanges.

2. Friction between circumferential helix release cord and the helix wire as the arms of the release mechanisms shift the release cord inside the antenna. As the antenna deploys and the release cord is fixed outside the antenna, there will be friction over the length of the helix release cord.

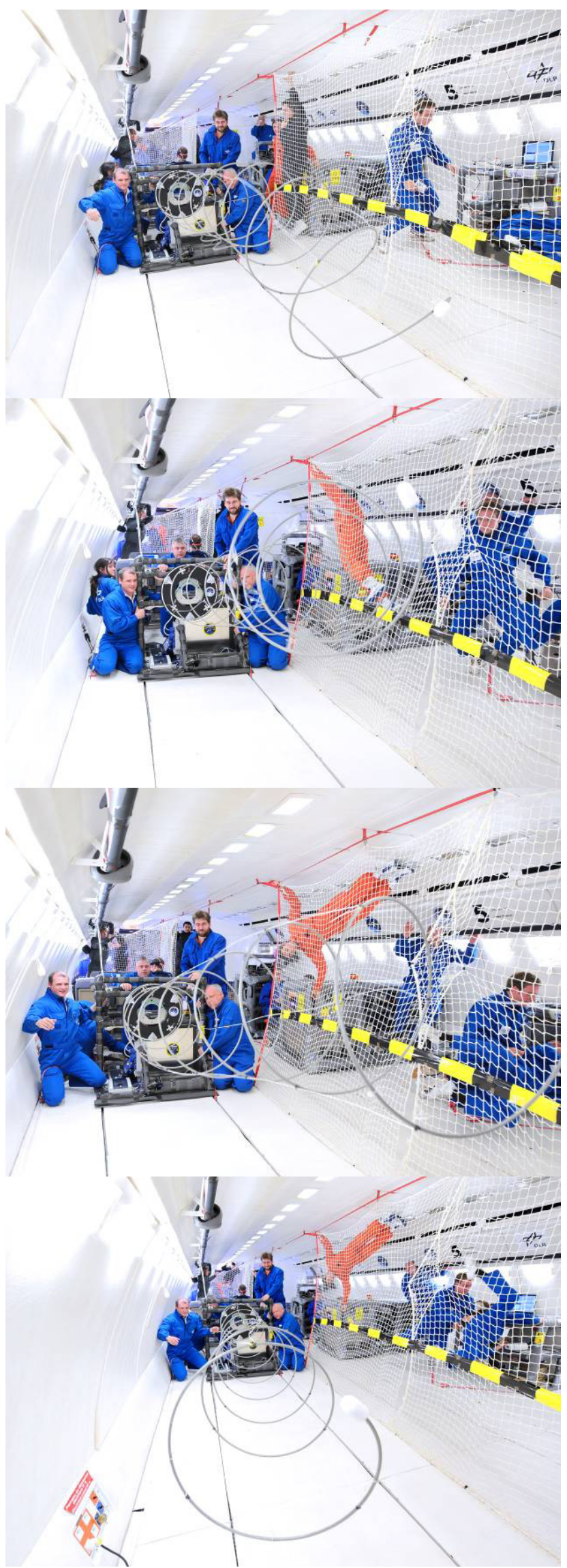

Figure 14 - Helix Antenna Deployment Sequence 
Already in pre-tests prior the PFC these effects could be noticed and eliminated to some extent. But design improvements are still mandatory.

A typical time-history of force transducer data for a deployment sequence (in parabola time) is given in Fig. 15. It shows the deployment of test structure P1 in parabola 1. Note: compression forces are positive in the diagram. Right after release $t_{0}$ there are compression forces coming from the inertia during deployment. A sharp peak is visible at $\mathrm{t}_{0}+1.1 \mathrm{sec}$, where the helix structure is abruptly stopped in its forward motion by the control cords. In the remaining $20 \mathrm{sec}$ until hyper-g the motion of the structure and hence the reaction forces cease noticeably. When entering the hyper-g phase at $t_{0}+22 \mathrm{sec}$ the structure is pushed against the aircraft floor causing again compression forces in this specific force transducer.

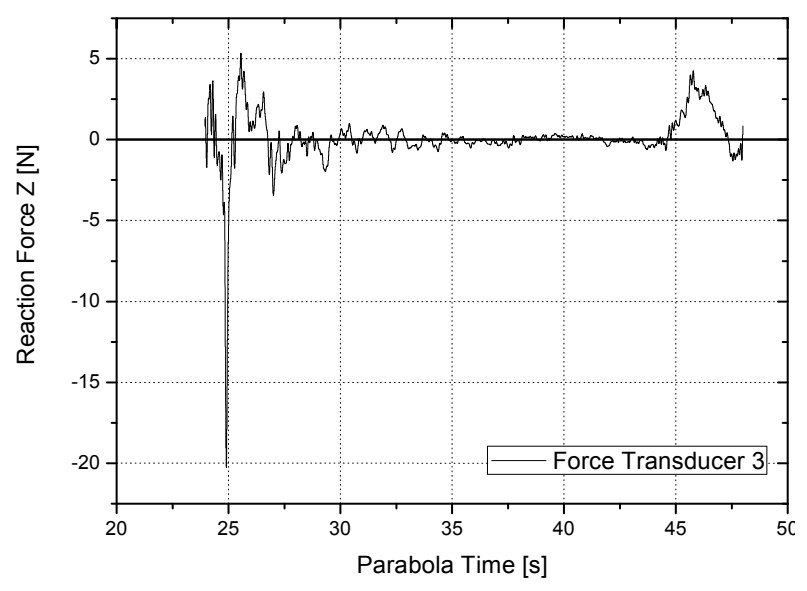

Figure 15 - Force-Time-History of a Deployment test

From the tests the deployment velocities were determined based on the force transducer data, as described above, and their correlation with the recorded video data. In all cases the deployment duration taken out of video and force transducer recordings matched very well.

The measured interface forces vary strongly depending on the zero-g environment. Fig. 15 gives an example of possible values for the test structure P1. Better values can not be given until the zero-g environment of the aircraft can be taken into account in the analysis of the data. This will be done by means of numerical analysis but is not concluded yet.

As expected the deployment velocity is dependent on the spring stiffness of the helix structure. For the given combinations of material and geometry it can be seen that the test structures with a wire diameter of $8.5 \mathrm{~mm}$ have lower deployment velocities than the structures with $9.5 \mathrm{~mm}$ wire diameter. In each group the CFRP structures have higher velocities because of the higher material stiffness. Values of the deployment velocities are listed in Table 2 hereafter. For the chosen combinations the lowest velocity for the $8.5 \mathrm{~mm}$ aramid structure $(\mathrm{P} 2)$ is $\approx 3.3 \mathrm{~m} / \mathrm{sec}$ and the highest for the $9.5 \mathrm{~mm}$ CFRP structure (P3) is $\approx 4.44 \mathrm{~m} / \mathrm{sec}$.

Besides the combinations of material and geometry the prestressing of the most favoured structure was changed. This was done by shortening the control cords by $500 \mathrm{~mm}$ leading to an overall structure length of $3.5 \mathrm{~m}$. The change in stiffness led to a slightly higher deployment velocity and hence structural stiffness. With this change the mechanical properties of the aramid structure with $9.5 \mathrm{~mm}$ diameter could be achieved. Hence, it could be shown that a higher pre-stressing is a good compromise of stiffness, favourable material properties (relaxation) and mass, even though just marginal in the existing case.

Table 2 - Deployment Time and Velocity

\begin{tabular}{c|c|r|r|}
\hline $\begin{array}{c}\text { Parabola } \\
\text { Sequence }\end{array}$ & Label & $\begin{array}{c}\text { Average Deploy } \\
\text { Time [sec] }\end{array}$ & $\begin{array}{c}\text { Average Deploy } \\
\text { Velocity [m/sec] }\end{array}$ \\
\hline 1 & P1 & $1.12 \mathrm{sec}$ & $3.58 \mathrm{~m} / \mathrm{sec}$ \\
\hline 2 & P2 & $1.20 \mathrm{sec}$ & $3.33 \mathrm{~m} / \mathrm{sec}$ \\
\hline 3 & P3 & $0.90 \mathrm{sec}$ & $4.44 \mathrm{~m} / \mathrm{sec}$ \\
\hline 4 & P4 & $1.08 \mathrm{sec}$ & $3.72 \mathrm{~m} / \mathrm{sec}$ \\
\hline 5 & P1 & $0.94 \mathrm{sec}$ & $3.73 \mathrm{~m} / \mathrm{sec}$ \\
\hline
\end{tabular}

As an outcome of the performed tests and their results it became obvious that the CFRP structure with the smaller diameter but a higher pre-stressing would perform best on the flight model of the AISat.

\section{DEPLOYMENT NuMERICAl Simulation}

In order to further optimize the mechanical antenna performance numerical analysis are performed. They will focus on the influence of the pre-stressing on the deployment velocity and the bending eigenfrequencies of the helix structure. The test data will be used for the model correlation. In a first step material parameters and damping values will, in reasonable ranges, be adjusted such that the deployment velocities of simulation and test match. In future investigations the correlation shall be extended to torsion and bending eigenmodes. Since torsional effects can be investigated even under gravity, these eigenfrequencies and damping values could already be determined. Fig. 16 shows a typical decay curve wrt. torsional vibrations. Concerning the correlation of the bending behavior, this can only be done by using the test results combined with the aircraft zero-g data in the numerical simulation and requires an already adjusted FE-model.

Since the whole deployment process is a highly non-linear problem in terms of geometry (very large deformations) and material (non-linear spring behavior of control cords) LSDyna was selected as numerical solver. It has a very good 
performance for the solving of non-linear problems either implicit or explicit. Furthermore, it offers all necessary features and suitable elements which are well suited for the existing problem.

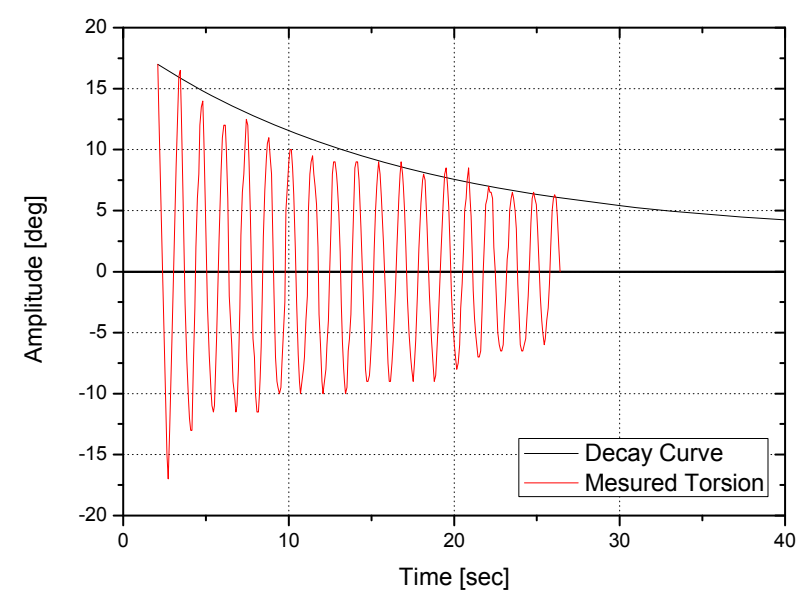

Figure 16 - Decay Curve of Torsion Motion

\section{Analysis Strategy}

In order to simulate the process of stowing and releasing the pre-stressed helix structure the analysis is split into two major parts:

1. The helix structure will be pre-stressed: explicit nonlinear static analysis

2. The helix structure will be stowed to a height of approx. $100 \mathrm{~mm}$ and released: implicit nonlinear transient analysis

In the first step the helix structure will be compressed from its manufacturing length to the required $4000 \mathrm{~mm}$ nominal length. At the end of this calculation the element stresses are output and used as input for the subsequent explicit analysis. The stress data are transferred to a complete FE model with nominal length, interface plate and control cords. This model is further compressed to the stowing height of $100 \mathrm{~mm}$, will be released and finally deploy.

Expected results from the analysis are interface forces during deployment and during decay, deflections of the antenna and the possibility of the derivation of eigenfrequency values from the calculated time history.

\section{FE-Model Description}

The overall FE model (see Fig. 17) consists of the ground plate (black), the helix structure (blue), the control cords (black) and the compression plate (green). Both ground plate and compression plate are modelled as rigid bodies (shell_el_form2). The ground plate itself has displacement boundary conditions on three points which resemble the position of the force transducers and hence the connection of the test structure to the test rack.

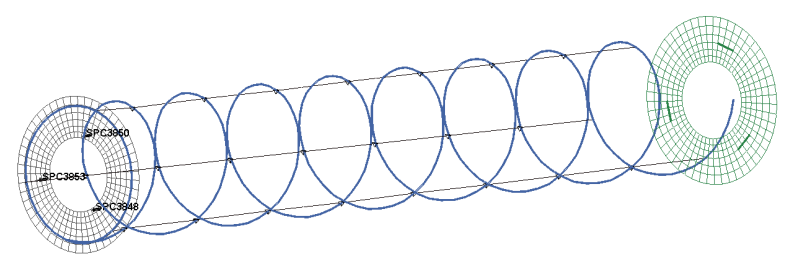

Figure 17 - Helix Antenna Finite Element Model

In order to keep the model size and calculation effort in the beginning as small as possible, the model of the helix is created from beam elements. This means, there is no volume representation of the sandwich tube. All necessary data are input as material properties. Since the helix material is anisotropic a special element formulation (beam_el_form2) had to be used to decouple Young's Modulus from Shear-Modulus via the rigidity terms and not just define an isotropic material. It allows the definition of the stiffness terms:

$\begin{array}{ll}\text { - bending: } & E I_{b} \\ \text { - torsion: } & G I_{t}\end{array}$

with $I_{b}$ being bending rigidity and $I_{t}$ being the torsional rigidity. Young's Modulus and Shear Modulus are still linked through the Poisson's Ratio. In the analysis the rigidity terms are adjusted to correlate the analysis results with test results.

The control cords are modeled using beam elements (el_seatbelt). They are fixed to the top and bottom winding. At the windings in between a contact formulation (cable_guide) allows a longitudinal relative motion between helix and control cords. Since the control cords are made from braided material they show a non-linear stiffness behavior. Therefore a load-strain-curve has been determined from a small test series.

A constant damping is globally defined to the model and will be adjusted according to the decay determined from test results.

\section{Qualitative Analysis Results}

First outcomes of the FE calculations are shown in Fig. 18. On the left side the stowing process is depicted. The uneven stowing of the structure as it can be seen in the third step of the stowing comes from displacement boundary conditions which have been set to avoid buckling of the helix during compression. Theses constraints will be switched off prior deployment. 
During the deployment it can be seen that the structure tends to make large sideward deflections even without disturbing factors like the before mentioned interaction between helix and mechanisms and the helix release cord itself. Furthermore it can be seen how the control cords tighten as the structure reaches full length and as they become loose as soon as the structure starts its backward motion.

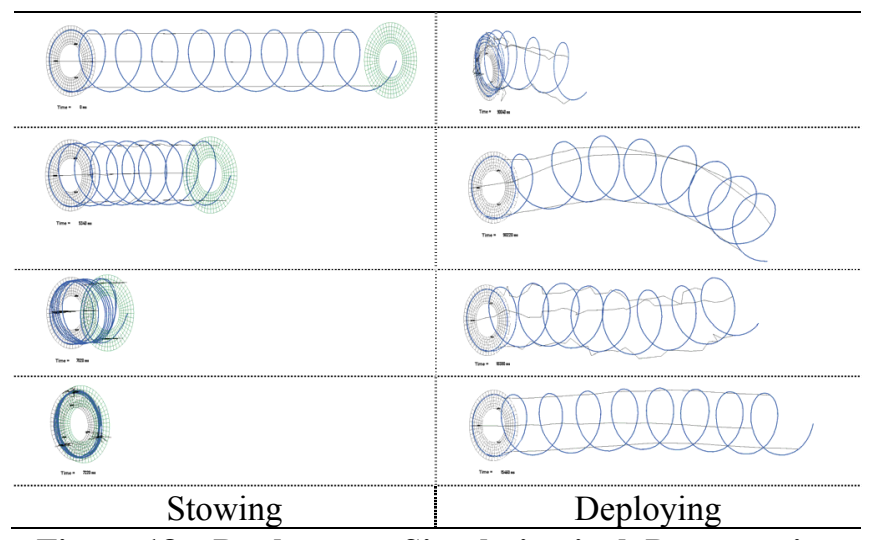

Figure 18 - Deployment Simulation incl. Pre-stressing

Force time histories can not be provided in this paper since there are still numerical problems which lead to a non-zero force balance in the analysis. Reasons for that are still under investigation.

\section{SUMMARY / CONCLUSIONS}

Although the complete evaluation of the parabolic flight tests had not yet been finalized in January 2011, the most decisive conclusions and preliminary results are already available:

The concept of a helical antenna which can be stowed and then, upon release, expand like a compression spring, is feasible.

With a stiffness reserve that is large enough to compensate losses induced by material creep and relaxation, and with suited control cords which limit the expansion, the helices will achieve a stable final length which can be adjusted in accordance with the electromagnetic requirements.

The direction into which the antenna moves forward immediately after release depends strongly on the uniformity of its interaction with the release mechanisms and the interaction with the helix release cord. High-quality release mechanisms with little friction will help to make the expansion as straight as possible.

The vibrations induced by the expansion and, in particular, by rebounds of the antenna tip when the control cords are tightened, decreased noticeably, although there was not sufficient time during the zero-g phases and too many mechanical disturbances from the aircraft to bring the antenna to a motionless end position.

The overall performance of carbonfiber-based antenna cores is superior to competing types. Consequently the AISat flight model will be equipped with such an antenna.

The recalculation of the antenna deployment using FEanalysis is ongoing. As soon as the test and analysis data could be correlated a further optimization of the antenna deployment behavior for future applications will be started.

\section{REFERENCES}

[1] King H.E., Wong J.L, Newman, E.H, Helical Antennas, in: Volakis J.L. (Ed.), Antenna Engineering Handbook, McGraw-Hill, New York 2007

[2] Balanis C.A., Modern Antenna Handbook, Wiley, Hoboken NJ 2008

[3] Robinson (Nov. 2, 1999). United States Patent 5.977.932 "Self-Deploying Helical Structure"

[4] Holemans. United States Patent 5.721.558 „Deployable Helical Antenna" (Feb. 24, 1998)

[5] Owen (Oct. 14, 1975). United States Patent 3.913.109 "Antenna Erection Mechanism"

[6] Heinemann et al (Jun. 1, 1999). United States Patent 5.909.197 "Deployable Helical Antenna Stowage in a Compact Retracted Configuration"

[7] Kurland et al (Sept. 17, 1974). United States Patent 3.836.979 "Lightweight Deployable Helical Antenna"

[8] Acker (Jan. 10, 1978). United States Patent 4.068.238 "Elastic Strain Energy Deployable Helical Antenna"

[9] Web source (17.11.2010): http://www.siltex.de/en/products.php?idProduct=19

[10] Schütze R., Lightweight Structures based on CFRP Sandwich Struts and CFRP Connections. Proceed. Internat. Symp. on Advanced Materials for Lightweight Structures, Noordwijk, March 1994 (ESA-WPP-070)

[11] Web source (17.11.2010): http://www.toraycfa.com/pdfs/T300DataSheet.pdf

[12] Web source (17.11.2010) http://www.vetrotexeurope.com/yarns.html\#04

[13] Web source (17.11.2010) http://www.mgs-online.com/de/techinfo/ti frame.htm 


\section{BIOGRAPHY}

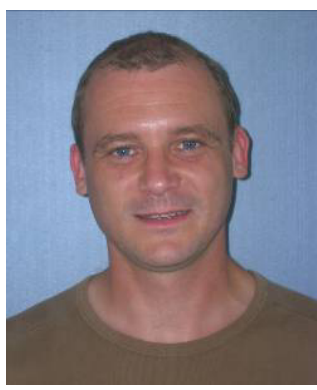

Tom Sproewitz is deputy head of the department of System Analysis - Space Segments of the DLR Institute of Space Systems since 2009 and in parallel leader of an internal NanoSatellite Project with launch in August 2011. In 2001 he graduated at the Technical University Chemnitz, Germany with a Diploma in Applied Mechanics as part of Mechanical Engineering. After graduation he worked at the DLR Institute of Composite Structures and Adaptive Systems as mechanical engineer. From 1998 to 2003, he was involved in the development, manufacturing and mechanical testing of the Rosetta-Lander Philae and the NetLander (end of Phase B) as Mechanical Analysis Engineer. Thermal analysis on porous sandwich structures for dish antennas on telecommunication satellites later became his field of competence. Since 2006, he has worked on structural design issues for aircraft components made from fiber composite materials and became deputy head of the Department of Composite Structures.

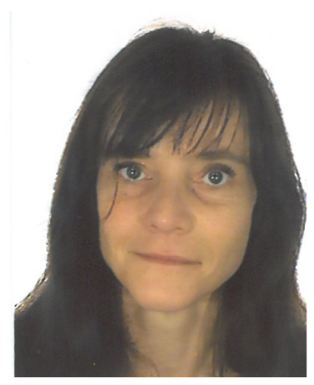

Annette Baeger graduated in 2000 from the Technical University of Berlin with a Diploma in Electrical Engineering. After graduation she worked as a freelancer in the fields of the development of measurement controlled manufacturing and development of test stands for automotive components. From 2005 she worked for the enterprise Astro- und Feinwerktechnik Adlershof $\mathrm{GmbH}$ in Berlin. There she was involved in mechanical environmental testing and quality assurance of space systems and components. Since 2009 Annette Bäger is laboratory head of the mechanical-dynamical test facilities within the Department System Conditioning of the DLR Institute of Space Systems. Here she accomplishes standard qualification tests for space systems, develops new test technologies and procedures and improves and optimizes the existing ones.

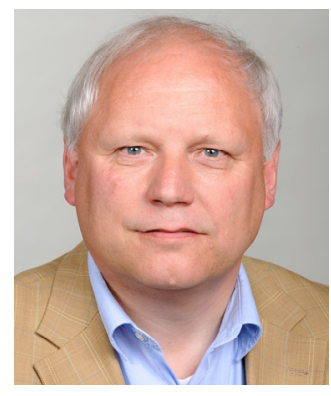

Prof. Dr. Joachim Block graduated with a diploma in Physics at the Technical University of Braunschweig, Germany, in 1981 and received his $\mathrm{PhD}$ degree at the University of Kassel, Germany, in 1988. His work was mainly focussed on physical measurement methods on lightweight space structures and on the adaptation of these methods to elevated temperatures. For more than 10 years from 1994 to 2004 he was responsible for development and qualification of the lander structure for ESA's cornerstone mission ROSETTA, which was launched in 2004. Since then he is responsible for the coordination of the scientific space projects performed by the DLR Institute of Composite Structures and Adaptive Systems in Braunschweig, Germany.

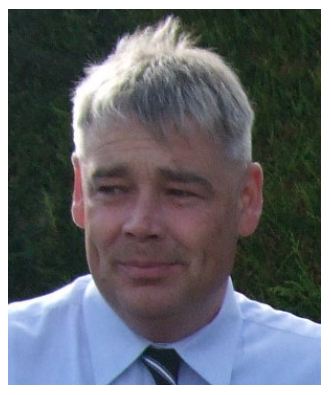

Lars-Christian Hauer is head of the Electronics Laboratory and leader of the AIS-project in the department of Orbital Systems of the DLR Institute of Space Systems since 2008. From 1988 to 1992 he made an apprenticeship as Radio and Telecommunication Craftsman and became Master Craftsman in 1997. From 1992 to 2004 he served as Master Chief Petty Officer in the German Navy where he was electronics technician for weapons and communication systems and worked as instructor for electronic reconditioning at the German Navy School. In 2005 he graduated at the University of Applied Sciences Wilhelmshaven with a Diploma in Electronic Engineering involving radiofrequency, microwave and antenna design. After his study from 2005 to 2008 he worked for the PZT-Testlab GmbH Wilhelmshaven as Electronics Test Engineer.

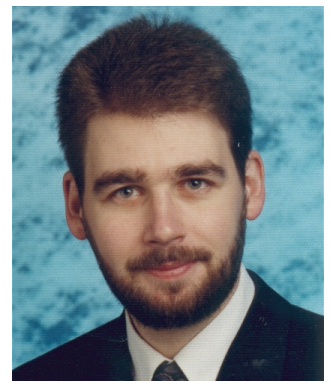

Dr. Martin Schuetze received his $\mathrm{Ph} . \mathrm{D}$. in Computer Science from the University of Kaiserslautern, Germany, in 1999. He then worked in aerospace and automotive industry where he was responsible for embedded hard- and software development teams. Currently, he is one of the directors of Schütze $\mathrm{GmbH} \& \mathrm{Co} \mathrm{KG}$, a German company developing and producing CFRP components for aerospace as well as general industrial applications. 
